\title{
Numerical simulation of astrophysical cyclotron-maser emission
}

\author{
D.C. Speirs, S.L. McConville, K.M. Gillespie, A.D.R. Phelps, A.W. Cross and K. Ronald \\ Department of Physics, SUPA, University of Strathclyde, Glasgow, G4 0NG, Scotland, UK.
}

\begin{abstract}
Numerical simulations have been conducted at the University of Strathclyde to study the spatial growth rate and emission topology of the cyclotron maser instability responsible for auroral magnetospheric radio emission from stars and planets and intense non-thermal radio emission in other astrophysical contexts. The results have significant bearing on the radiation propagation characteristics and highly debated question of escape from the source region.
\end{abstract}

\section{INTRODUCTION AND BACKGROUND}

A variety of astrophysical radio emissions have been identified to date associated with non-uniform magnetic fields and accelerated particle streams [1]. Numerous such sources, including planetary and stellar auroral radio emission are spectrally well defined with a high degree of extraordinary (X-mode) polarisation. In particular, for the terrestrial auroral case it is now widely accepted that such emissions are generated by an electron cyclotron-maser instability driven by a horseshoe shaped electron velocity distribution [2-5]. Such distributions are formed when particles descend into the increasing magnetic field of planetary/stellar auroral magnetospheres, where conservation of magnetic moment results in the conversion of axial momentum into rotational momentum forming an electron velocity distribution having a large spread in pitch factor. Theory has shown that such distributions are unstable to cyclotron emission in the X-mode [6] and inhomogeneous systems have also been examined [7].

Experiments and simulations carried out at the University of Strathclyde [8-16] have investigated the electrodynamics of an electron beam subject to significant magnetic compression. Analytical theory [3-7,17] at the University of St Andrews and at the STFC, Rutherford Appleton Laboratory has greatly benefitted this experimental and numerical simulation research. More recently, PiC simulations have been conducted to study the cyclotron-maser emission topology in the absence of radiation boundaries and as a function of various beam parameters, with the spatial growth of the instability and characteristics of radiation propagation documented.

\section{RESULTS}

In the initial scaled laboratory experiments conducted at Strathclyde, an electron beam was injected from an annular explosive emission cathode and subject to significant magnetic compression using a highly configurable solenoid arrangement. The beam transport characteristics and cyclotronresonant beam-wave interaction within a circular cylindrical waveguide were simulated in $2.5 \mathrm{D}$ using the $\mathrm{PiC}$ code KARAT, with a typical electron beam trajectory presented in figure 1 for a magnetic mirror ratio of $B_{z} / B_{z 0}=34$ and peak magnetic flux density of $0.487 \mathrm{~T}$. There is clear evidence of pitch angle expansion within the plotted trajectory, as indicated by the presence of a radially diffuse mirrored component within the convergent magnetic field region.



Fig. 1. Overview of experimentally consistent, waveguidebounded PiC simulation geometry.

Within both the waveguide bounded simulations and experiments, cyclotron resonant energy transfer was studied with near-cutoff Transverse Electric (TE) modes, which closely match the electromagnetic field polarization of the Xmode with respect to the magnetostatic field. Although radiation propagation and coupling was assumed to be in the forward-wave direction (with respect to the cathode), a significant number of cyclotron-wave detunings resulted in backward-wave coupling. An example of such a regime is presented in figure 2, where a contour plot of $\mathrm{E}_{\theta}$ from a 2.5D PiC simulation shows a more prominent backward-wave character to the cyclotron-wave coupling.

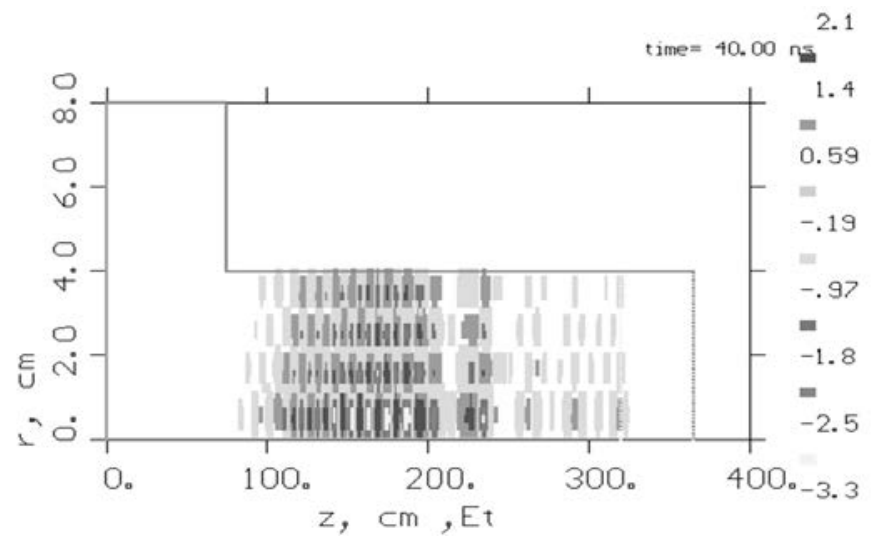

Fig. 2. Contour plot of $\mathrm{E}_{\theta}$ within a waveguide-bounded simulation geometry. 
In order to bridge between the waveguide-bounded experiments and an unbounded astrophysical scenario, we conducted PiC simulations to study the horseshoe cyclotronmaser instability in the absence of radiation boundaries. For the purpose of simulating the unbounded interaction geometry, a $44 \mathrm{~cm}$ thick region of radially increasing conductivity was defined around the $4 \mathrm{~m}$ propagation path of a $20 \mathrm{keV}, 14 \mathrm{~A}$ electron beam. This represented an idealised absorber of electromagnetic radiation, inhibiting reflection and the formation of boundary resonant eigenmodes. Other simulation parameters include a predefined electron beam horseshoe distribution, comprising a pitch spread of $\alpha=\mathrm{v}_{\perp} / \mathrm{v}_{\mathrm{z}}=0 \rightarrow 9.5$ and a uniform axial magnetic flux density of $0.1 \mathrm{~T}$.



Fig. 3. 3D contour plot of $E_{\theta}$ within an unbounded simulation geometry.

The simulations demonstrate a clear relaxation of the electron horseshoe distribution via the cyclotron maser instability, with RF output generated in the X-mode at the electron cyclotron frequency and with a small negative axial wavenumber (fig 3). This is evidenced by the oblique propagation angle of the wavefront highlighted in figure 3 , which factoring in the differing spatial scales of the $z$ and $r$ axes corresponds to a backward-wave propagation angle of a few degrees.

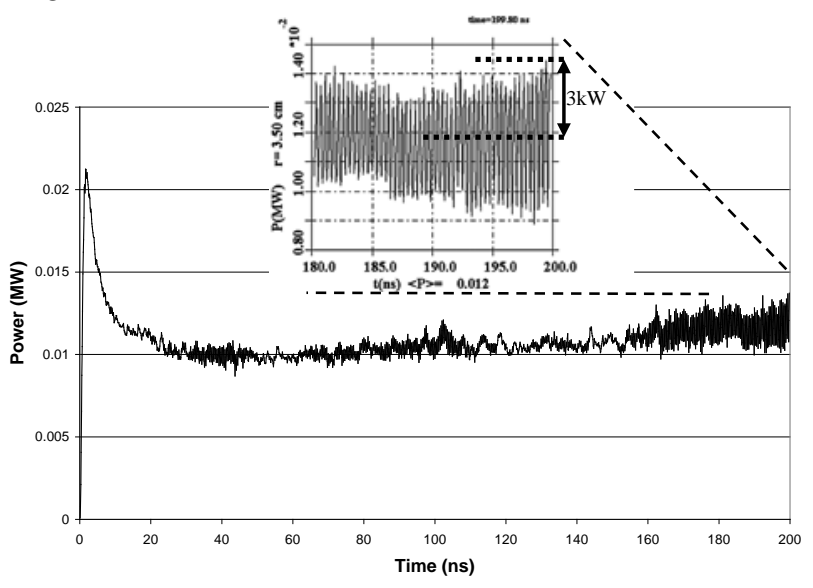

Fig. 4. Radial Poynting flux temporal evolution, measured in a plane at $r=3.5 \mathrm{~cm}$ and spanning the length of the simulation.
A plot of the corresponding radial Poynting flux is presented in figure 4 measured in a plane at $\mathrm{r}=3.5 \mathrm{~cm}$. A DC offset is present in the measurement due to low frequency EM field components associated with the electron beam propagation. After $180 \mathrm{~ns}$ a peak rf output power of $\sim 3 \mathrm{~kW}$ was measured corresponding to an rf conversion efficiency of $1.1 \%$ - comparable to the estimate of $\sim 1 \%$ for the astrophysical phenomena [19].

\section{CONCLUSION}

PiC simulations have been conducted to study the electrodynamics and emission topology of the cyclotron maser instability responsible for auroral magnetospheric radio emission and the generation of non-thermal radio emission in other astrophysical contexts [20]. Initial waveguide-bounded PiC simulations and laboratory experiments show for numerous near-cutoff resonance regimes, a tendency for cyclotron-wave coupling to occur with a backward-wave character. This trend is reflected in the results of unbounded PiC simulations of cyclotron-wave coupling, where radiation propagation has been observed with a distinctive backwardwave character to it, typically a few degrees from perpendicular to the magnetostatic field. The corresponding RF conversion efficiency of $1.1 \%$ in the unbounded case is comparable to earlier waveguide bounded simulations [12] and consistent with estimates of $\sim 1 \%$ for the astrophysical phenomena [18-20].

\section{ACKNOWLEDGMENT}

The authors would like to thank the EPSRC for funding and Prof. V.P. Tarakanov for his assistance with the PiC code KARAT.

\section{REFERENCES}

[1] Zarka P., 1992, Advances in Space Research, 12, pp. 99-115.

[2] Ergun R.E., et al., 2000, Astrophys. J., 538, pp. 456-466.

[3] Bingham R. and Cairns R.A., 2000, Phys. Plasmas, 7, pp. 3089-3092.

[4] Bingham R., et al., 2004, Contrib. Plasma Phys., 44, pp. 382-387.

[5] Cairns R.A., et al., 2005, Physica Scripta, T116, pp.23-26.

[6] Bingham R., Cairns R.A. and Kellett B.J., 2001, Astron. Astrophys., 370, pp. 1000-1003.

[7] Cairns R.A., et al., 2011, Phys. Plasmas, 18, art. 022902.

[8] Speirs D.C., et al., 2005, J. Plasma Phys., 71, pp. 665-674.

[9] McConville S.L., et al., 2008, Plasma Phys. Control. Fusion, 50, art. 074010.

[10] Ronald K., et al., 2008, Plasma Sources Sci. Technol., 17, art. 035011.

[11] Ronald K et al., 2008, Phys. Plasmas, 15, art. 056503.

[12] Speirs D.C. et al., 2008, Plasma Phys. Control. Fusion, 50, art.074011.

[13] Ronald K., et al., 2011, Plasma Phys. Control. Fusion, 53, art. 074015.

[14] McConville S.L. et al., 2011, Plasma Phys. Control. Fusion, 53, art. 124020.

[15] Speirs D.C., et al., 2010, Phys. Plasmas, 17, art. 056501.

[16] Gillespie K.M., et al., 2008, Plasma Phys. Control. Fusion, 50, art. 124038.

[17] Vorgul I., et al., 2011, Phys. Plasmas, 18, art. 056501.

[18] Bingham R., et al., 2013, Space Science Reviews, 10.1007/s11214-0139963-z.

[19] Gurnett D.A., et al., 1974, J. Geophys. Res., 79, pp. 4227-4238.

[20] Treumann R.A., 2006, Astron. Astrophys. Rev., 13, pp. 229-315. 